\title{
ACTITUDES HACIA LAS PERSONAS CON DISCAPACIDAD EN UNA MUESTRA DE ESTUDIANTES DE EDUCACIÓN SECUNDARIA DE LIMA
}

\section{Attitudes toward people with disabilities in a sample of students of high school of Lima}

Sergio Domínguez Lara*

\section{Resumen}

Objetivo: determinar si existen diferencias en cuanto a las actitudes hacia las personas con discapacidad en una muestra de estudiantes de educación secundaria de Lima, considerando variables tales como sexo, contacto con personas con discapacidad y tipo de colegio. Método: Diseño ex post facto de tipo retrospectivo y tipo descriptivo-comparativo. Se evaluó a 432 estudiantes de 4to y 5to año de educación secundaria de colegios estatales y privados de Lima Metropolitana, de los cuales 237 fueron varones (50.9\%) y 195 mujeres (45.1\%) a través de una Escala de Actitudes hacia las Personas con Discapacidad. Resultados: se reportan diferencias significativas entre varones y mujeres con relación a las escalas Reconocimiento/Negación de Derechos $(t(430)=-3.111 ; p<.01 ; d$ de Cohen $=.30)$ e Implicación Personal $(t(430)=-5.438 ; p<.001 ; d$ de Cohen=.53), a favor de las adolescentes; con relación al tipo de colegio en Valoración de Capacidades y Limitaciones $(t(430)=4.151 ; p<.001 ; d$ de Cohen $=.40)$, Calificación Genérica $(t(430)=$ 2.336; $p<.05 ; d$ de Cohen =.23) y Asunción de Roles $(t(430)=3.582 ; p<.001 ; d$ de Cohen =.34) a favor de los colegios particulares. Por último, con relación al contacto con personas con discapacidad, existen diferencias en la escala Implicación Personal $(t(430)=2.585 ; p<.01 ; d$ de Cohen =.26) a favor de las personas que presentan algún contacto. Conclusión: Los hallazgos servirán de base para realizar propuestas dirigidas a optimizar las actitudes, y potenciar las que estén favoreciendo a la integración de las personas con discapacidad.

Palabras clave: Actitudes, discapacidad, adolescencia.

\begin{abstract}
Objective: To determine if there are differences in attitudes towards people with disabilities in a sample of high school students in Lima, considering variables such as sex, contact with people with disabilities and type of school. Method: Design ex post facto retrospective and descriptive-comparative. Were evaluated 432 students of high school from state and private schools of Lima, 237 males (50.9\%) and 195 women (45.1\%). Was used the Scale of Attitudes toward Individuals with Disability. Results: We report significant differences between men and women in relation to the scales Recognition/ Denial of Rights $(t(430)=-3.111 ; p<.01 ; d$ de Cohen $=.30)$ and Personal Implication $(t(430)=-5.438 ; p<.001 ; d d e$ Cohen $=.53)$ in favor of women; in relation to the type of school, in Valuing Capabilities and Limitations $(t(430)=4.151$; $p<.001 ; d$ de Cohen =.40), Generic Description $(t(430)=2.336 ; p<.05 ; d$ de Cohen =.23) and Assumption of Roles $(t(430)=3.582 ; p<.001 ; d$ de Cohen $=.34)$ in favor of private schools. Finally, regarding the contact with people with disabilities, there are differences in the scale Personal Implication $(t(430)=2.585 ; p<.01 ; d$ de Cohen $=.26)$ for people who have some contact. Conclusion: The findings provide the basis for proposals to optimize the attitudes, and empower those who are promoting the integration of people with disabilities.
\end{abstract}

Keywords: Attitude, disability, adolescents.

* Docente investigador de la Facultad de Psicología y Trabajo Social de la Universidad Inca Garcilaso de la Vega. sdominguezmpcs@gmail.com 


\section{INTRODUCCIÓN}

De acuerdo con la Organización Mundial de la Salud (2001), la discapacidad hace referencia a una interacción multidireccional entre la persona y el contexto socioambiental en el que se desenvuelve, es decir, entre las funciones y estructuras alteradas del cuerpo, la capacidad para realizar diversas actividades como persona, su participación o desempeño real en las mismas, y las interacciones con los factores tanto personales como ambientales, que pueden actuar como barreras y ayudas.

Complementando aquello, Verdugo (2005) plantea que debe ser entendida de tres modos: como las deficiencias que puede tener una persona en su cuerpo, como a las limitaciones en sus actividades cotidianas $\mathrm{y}$, finalmente, como las restricciones que puede tener en su funcionamiento social como consecuencia de dicha deficiencia. Las deficiencias orgánicas, las deficiencias funcionales de actividad y las restricciones de participación social son los tres aspectos a tener en cuenta a la hora de entender la discapacidad y definirla.

A partir de aquello, la discapacidad debe comprenderse desde una perspectiva contextualista, con apoyo de un modelo ecológico de discapacidad, el cual debe estar basado en la interacción del individuo con su contexto (Schalock \& Verdugo, 2003), en el que se plantea que la discapacidad no es algo fijo ni dicotomizado; sino un proceso fluido, continuo y cambiante, y que además depende tanto de las limitaciones funcionales de la persona como de los apoyos disponibles en su entorno.

Estos planteamientos rescatan un elemento crucial que es la interacción de la persona con discapacidad con el entorno del cual forma parte, ya que si este no le brinda los apoyos necesarios, toda intención de integración e inclusión, será en vano. Esto quiere decir, siguiendo a Oliver (1990), que las restricciones impuestas a las personas con discapacidad no son una consecuencia directa de su deficiencia sino producto del ambiente social que no considera su desarrollo como un aspecto esencial.

Es por ello que es importante conocer cuales son las actitudes que existen en la población respecto a las personas con discapacidad, en tanto más adelante aquella les brindarán las oportunidades de integración social que les corresponden y que son necesarias (Moreno, Rodríguez, Saldaña \& Aguilera, 2006).

Las actitudes negativas constituyen una de las principales barreras para la inclusión e integración de las personas con discapacidad (Antonak \& Livneh, 2000; Polo, Fernández \& Díaz, 2011), y es por ello que siguen siendo motivo de análisis, ya que pueden facilitar la formación de prejuicios y estereotipos que las estigmatizan y excluyen, lo que tiene un efecto negativo en su calidad de vida (Shriver, 2003, Vignes et al., 2009). Se puede decir entonces que las actitudes negativas hacia las personas con discapacidad obstaculizan el desempeño de los roles y el logro de los objetivos vitales que se plantean las personas con discapacidad (Antonak \& Livneh, 2000; SafiliosRothschild, 1976).

El romper las barreras físicas, sociales y actitudinales existentes contra las personas con discapacidad es tan importante, tanto más que curar las deficiencias físicas o mentales (Imrie, 1997), se ha hallado que en pocos lugares se implementan actividades dirigidas a evaluar y mejorar las actitudes (Verdugo, Jenaro \& Arias, 1995), aún sabiendo que si se modifican las actitudes se favorecería una situación de igualdad y una integración social efectiva de las personas con discapacidad (Verdugo \& Arias, 1991).

En tal sentido, es importante conocer las actitudes hacia las personas con discapacidad por parte de los escolares debido a que en el contexto actual de inclusión educativa, pueden facilitar la integración de los escolares con algún tipo de discapacidad, ya que el solo acceso de la personas con discapacidad a la escuela no es una garantía de participación total debido a las actitudes negativas de sus pares (Vignes et al., 2009), destacándose que la escuela es un elemento importante para la preparación de cara al empleo, la independencia y la participación en el contexto (Verdugo, 2003).

Además de ello, trabajar con escolares brinda un panorama más alentador en torno a una posible sensibilización en el tema, dado que aquellos elementos que se integran en su personalidad, incluyendo las actitudes, se hallan en configuración (Muratori, Guntín 
\& Delfino, 2010) y son susceptibles de cambio, lo cual podría enfocarse dentro de la prevención, dado que en la vida adulta resulta más difícil modificar actitudes (Ashman, 1982, en Vera, 1995). De acuerdo con Verdugo (2003), el desarrollo de la integración escolar contribuye a transformar las actitudes sociales y profesionales hacia la población más desfavorecida.

Con relación a estudios con niños y adolescentes, Talou, Borzi, Sánchez, Iglesias \& Hernández (2008/2009) realizarón una revisión de estudios llevados a cabo en Argentina con relación a las concepciones acerca de las personas con discapacidad en niños y adolescentes escolares de 6 a 15 años de edad. Encontraron que los estudiantes recibían escasa información sobre la problemática de las niñas y los niños con diversidad funcional. Inclusive, en algunos casos no mencionaban la existencia de compañeros con discapacidad en sus aulas, aún cuando sus escuelas estaban bajo planes de inclusión educativa.

Por su parte, Vignes et al. (2009) contaron con la participación de 1135 escolares franceses, 612 mujeres y 523 varones, de edades comprendidas entre 10 años 8 meses y 15 años. Usaron para tal fin el ChedokeMcMaster Attitudes Towards Children with Handicaps $(\mathrm{CATCH})$, el cual evalúa los componentes afectivo, cognitivo y conductual de las actitudes. De acuerdo con lo reportado por los autores, un incremento de las puntuaciones en el instrumento se asocia con conocimiento de discapacidad, tener un familiar cercano con discapacidad y contar con un amigo con discapacidad. Por otro lado, el componente cognitivo obtuvo puntuaciones más baja que los componentes afectivo y conductual en la muestra estudiada. Asimismo, son las mujeres quienes presentan actitudes más favorables, con relación a los varones.

En Latinoamérica, Muratori, Guntín \& Delfino (2010) estudiaron las actitudes de adolescentes argentinos hacia las personas con discapacidad. Participaron en ese estudio 265 adolescentes, de los cuales el $70.6 \%$ eran mujeres y el $29.4 \%$ eran varones. Sus edades oscilaron entre 15 y 19 años (Media=16.22). Fueron usados un cuestionario sociodemográfico y la Escala de Actitudes hacia las Personas con Discapacidad (Verdugo, Jenaro \& Arias, 1995). Los autores refieren que los escolares presentan, en términos generales, una actitud favorable hacia las personas con discapacidad. Por su parte, aquellos escolares que tienen algún tipo de contacto con personas con discapacidad generan implicación personal, asî como ideas desprovistas de estereotipos negativos. Cabe resaltar que no se efectuaron medidas del tamaño del efecto, por lo cual las comparaciones no deben ser tomadas como concluyentes. Refieren además una relación positiva y estadísticamente significativa entre frecuencia de contacto y las actitudes ("Valoración de Limitaciones y Capacidades" y "Reconocimiento/ Negación de Derechos"), aunque dichas correlaciones resultan bastante bajas (.17 y .19) para determinar una significación práctica de los hallazgos. Con relación al sexo, las mujeres presentan actitudes más favorables que sus pares varones (reconocimiento de derechos, implicación personal, calificación genérica). Al igual que las comparaciones anteriores, sería conveniente efectuar una medida del tamaño del efecto.

Por otro lado, García \& Hernández (2011) analizaron las actitudes que presentan los jóvenes y adultos de Chiapas (México) hacia las personas con discapacidad. Trabajaron con 736 personas, 341 y 395 mujeres. La edad oscila entre 13 y 83 años, siendo el grupo con más personas el de 20 a 30 años (36.4\%), seguido del de 31 a 40 años (22.3\%). Usaron la Escala de Actitudes hacia las Personas con Discapacidad (Verdugo, Jenaro \& Arias, 1995). Consideran variables como sexo, edad, estado civil, escolaridad, ocupación y religión. Asimismo, también se consideró el tipo de contacto que tiene el encuestado con la persona con discapacidad. Se encontraron actitudes favorables con relación al derecho a casarse y divertirse como las demás personas, aunque actitudes desfavorables relacionadas con la segregación e infantilización de sus comportamientos. Se presentaron actitudes más favorables entre aquellos que tienen un familiar con discapacidad y en aquellos cuyo contacto con personas con discapacidad es esporádico. No se hallaron diferencias estadísticamente significativas respecto al sexo ni religión.

Es así que para poder intervenir en esta realidad es necesario conocerla. En tal sentido, el objetivo del presente trabajo fue describir las actitudes de los escolares limeños hacia las personas con discapacidad en función de variables tales como sexo, contacto con personas con discapacidad y tipo de colegio. 


\section{METODO}

Se usó un diseño ex post facto de tipo retrospectivo (Montero \& León, 2007). Asimismo, es una investigación de tipo descriptivo-comparativa (Hernández, Fernández \& Baptista, 2003).

\section{Sujetos}

Se consideró una muestra intencional de 432 estudiantes de 4to y 5to año de educación secundaria de colegios estatales y privados de Lima Metropolitana, de los cuales 237 son varones (50.9\%) y 195 mujeres (45.1\%), con edades comprendidas entre 13 y 18 años (Media 15.34 años). El muestreo utilizado fue de tipo intencional.

Tabla 1

Características sociodemográficas de la muestra

\begin{tabular}{lcc}
\hline Sexo & Frecuencia & Porcentaje \\
\hline Masculino & 237 & 54.9 \\
Femenino & 195 & 45.1 \\
& & \\
Edad & 2 & .5 \\
13 & 57 & 13.2 \\
14 & 191 & 44.2 \\
15 & 151 & 35.0 \\
16 & 29 & 6.7 \\
17 & 2 & .5 \\
18 & & \\
Año de estudios & 221 & 51.2 \\
4to año & 211 & 48.8 \\
5to año & & \\
Gestión del colegio & & \\
& & \\
Particular & 239 & 55.3 \\
Estatal & 193 & 44.7 \\
¿Contacto con PCD? & & \\
No & 271 & 62.7 \\
Sí & 161 & 37.3 \\
\hline
\end{tabular}

\section{Instrumento}

\section{Cuestionario sociodemográfico}

Se consideraron datos personales tales como: Edad, sexo, año de estudios, y además si presenta algún tipo de contacto con personas con discapacidad (Formato cerrado SI/NO). En caso afirmativo, señalaron la razón de contacto (Familiar, Laboral, Asistencial, Ociol Amistad y Otras razones), frecuencia de contacto (Muy frecuente, Frecuente, Poco frecuente y Casi nula), calidad de contacto (Muy Positivo, Positivo, Neutral, Negativo y Muy Negativo) y el tipo de discapacidad con la cual tienen contacto (Física, Auditiva, Visual, Retraso mental y Múltiple).

\section{Escala de Actitudes hacia las Personas con Discapacidad}

Fue desarrollada por Verdugo, Jenaro \& Arias (1994). Se trata de una escala multidimensional desarrollada en España, que cuenta con estudios de fiabilidad (alfa de Cronbach .92) y validez (una general y otras específicas para deficiencias físicas, sensoriales y mentales). En un estudio previo se confirmó su estructura pentafactorial, y se hallaron coeficientes de consistencia adecuados (Dominguez, Aquije, Castillo, Dulanto, Llontop \& Rimachi, en prensa).

La persona evaluada debe opinar si está de acuerdo o no con cada una de las frases que se presentan, formuladas positiva o negativamente, siendo los significados de las opiniones los siguientes: Estoy muy de acuerdo (MA); Estoy bastante de acuerdo (BA); Estoy parcialmente de acuerdo (PA); Estoy parcialmente en desacuerdo (PD); Estoy bastante en desacuerdo (BD); Estoy en total desacuerdo (TD).

La escala (Anexo 1), presenta dimensiones que se vinculan con la valoración de las capacidades y limitaciones de la persona con discapacidad; el reconocimiento o negación de derechos; los comportamientos concretos de interacción que la persona llevaría a cabo en relación con personas con discapacidad (implicación personal); las atribuciones globales y concepciones generales que se efectúan acerca de rasgos presuntamente definitorios de la personalidad o conducta de la persona con discapacidad (calificación genérica); y las presunciones del encuestado acerca de la concepción que de sí mismos 
tienen las personas con discapacidad (asunción de roles).

En el presente estudio obtuvo los siguientes coeficientes de consistencia interna (alfa de Cronbach):

Valoración de las capacidades y limitaciones: .703(IC al 95\%: .659: .743)

Reconocimiento o negación de derechos: .778 (IC al 95\%: .745: .808)

Implicación personal: .623 (IC al 95\%: .566: .675)

Calificación genérica: .510 (IC al 95\%: .433: .579)

Asunción de roles: .665 (IC al 95\%: .610: .714)

Escala Total: .878 (IC al 95\%: .861: .894)

\section{Procedimiento}

Se les informó a los participantes el objetivo de la investigación, y se garantizó la confidencialidad de los resultados. El instrumento fue administrado por un solo investigador, aclarándose las dudas de los estudiantes cuando ellos lo requerían.

Se elaboró una base de datos en MS Excel para ser analizada posteriormente. Se invirtieron reactivos que expresaban "actitudes negativas" (ítems 1, 2, 4, 5, 6, 7, $8,10,15,17,18,23,24,25,26,28,29,31,34,35$ y 37 ) de tal manera que todos los ítems apunten a una misma dirección y una puntuación elevada exprese una actitud favorable hacia la persona con discapacidad.

Para el análisis de comparación se conformaron los grupos en función del sexo, contacto con personas con discapacidad, tipo de colegio y año de estudios. Dicho análisis se realizó mediante la prueba $\mathrm{t}$ de Student, calculándose posteriormente el tamaño del efecto de tal comparación, a fin de comprobar la relevancia de la misma. Para el análisis de los datos se utilizó el paquete estadístico OPENSTAT (Miller, 2003) y un módulo de cálculo del Tamaño del Efecto (Dominguez, en prensa).

\section{RESULTADOS}

Tabla 2

Aspectos descriptivos de las subescalas

\begin{tabular}{lcc}
\hline Escalas & $\begin{array}{l}\text { Media } \\
\text { Aritmética }\end{array}$ & $\begin{array}{l}\text { Desviación } \\
\text { Estándar }\end{array}$ \\
\hline $\begin{array}{l}\text { Valoración de Capacidades y } \\
\text { Limitaciones }\end{array}$ & 43.07 & 6.55 \\
Reconocimiento/Negación de & 57.18 & 6.87 \\
Derechos & 35.84 & 4.59 \\
Implicación Personal & 20.94 & 3.63 \\
Calificación Genérica & 18.93 & 3.36 \\
Asunción de Roles & 175.96 & 19.07 \\
Escala Total & & \\
\hline
\end{tabular}

De acuerdo con los resultados hallados, todas las subescalas, incluida la escala total, se hallan a un nivel promedio (Dominguez et al., en prensa). En tal sentido, la distribución percentilar es la siguiente:

Valoración de Capacidades y Limitaciones: Pc 55 (Nivel Promedio)

Reconocimiento/Negación de Derechos: Pc 45 (Nivel Promedio)

Implicación Personal: Pc 50 (Nivel Promedio)

Calificación Genérica: Pc 60 (Nivel Promedio)

Asunción de Roles: Pc 55 (Nivel Promedio)

Escala Total: Pc 50 (Nivel Promedio)

Debe mencionarse que la subescala que destaca es la referida a Calificación Genérica (Pc 60), y la subescala de menor cuantía es la de Reconocimiento/ Negación de Derechos (Pc 45). 


\section{Actitudes hacia las personas con discapacidad según el sexo}

Tabla 3

Diferencias de acuerdo al sexo respecto a los factores de la Escala

\begin{tabular}{|c|c|c|c|c|c|}
\hline Factor & Sexo & Media & $\begin{array}{l}\text { Desv. } \\
\text { Estándar }\end{array}$ & $\mathbf{t}(430)$ & d de Cohen \\
\hline \multirow[t]{2}{*}{$\mathrm{F} 1$} & Varones & 42.85 & 6.49 & -.782 & .07 \\
\hline & Mujeres & 43.34 & 6.63 & & \\
\hline \multirow[t]{2}{*}{$\mathrm{F} 2$} & Varones & 56.26 & 7.15 & $-3.111 * *$ & .30 \\
\hline & Mujeres & 58.30 & 6.35 & & \\
\hline \multirow[t]{2}{*}{ F3 } & Varones & 34.78 & 4.79 & $-5.438 * * *$ & .53 \\
\hline & Mujeres & 37.12 & 3.98 & & \\
\hline \multirow[t]{2}{*}{$\mathrm{F} 4$} & Varones & 20.71 & 3.65 & -1.488 & .14 \\
\hline & Mujeres & 21.23 & 3.61 & & \\
\hline \multirow[t]{2}{*}{ F5 } & Varones & 18.96 & 3.24 & .246 & .02 \\
\hline & Mujeres & 18.88 & 3.51 & & \\
\hline \multirow[t]{2}{*}{ Total } & Varones & 173.56 & 19.84 & $-2.910 * *$ & .28 \\
\hline & Mujeres & 178.88 & 17.70 & & \\
\hline
\end{tabular}

$* \mathrm{p}<.05 ; * * \mathrm{p}<.01 ; * * * \mathrm{p}<.001$

De acuerdo con los resultados, las mujeres presentan, en términos generales, actitudes más favorables hacia las personas con discapacidad que los varones $(\mathrm{t}(430)=-2.910 ; \mathrm{p}<.01 ; \mathrm{d}$ de Cohen $=.07)$. Asimismo, difieren de forma significativa con relación a sus pares varones en cuanto al Reconocimiento/
Negación de Derechos $(\mathrm{t}(430)=-3.111 ; \mathrm{p}<.01 ; \mathrm{d}$ de Cohen $=.30)$ e Implicación Personal $(\mathrm{t}(430)=-5.438$; $\mathrm{p}<.001 ; \mathrm{d}$ de Cohen=.53). Con relación a las subescalas restantes, no se hallaron diferencias significativas con relación al sexo.

\section{Actitudes hacia las personas con discapacidad según el colegio de procedencia}

Tabla 4

Diferencias de acuerdo al colegio de procedencia respecto a los factores de la Escala

\begin{tabular}{|c|c|c|c|c|c|}
\hline Factor & Colegio & Media & $\begin{array}{l}\text { Desv. } \\
\text { Estándar }\end{array}$ & $t(430)$ & d de Cohen \\
\hline \multirow[t]{2}{*}{$\mathrm{F} 1$} & Particular & 44.23 & 6.59 & $4.151 * * *$ & .40 \\
\hline & Nacional & 41.64 & 6.23 & & \\
\hline \multirow[t]{2}{*}{$\mathrm{F} 2$} & Particular & 57.43 & 7.03 & .829 & .08 \\
\hline & Nacional & 56.88 & 6.67 & & \\
\hline \multirow[t]{2}{*}{ F3 } & Particular & 35.56 & 4.80 & -1.387 & .14 \\
\hline & Nacional & 36.18 & 4.30 & & \\
\hline \multirow[t]{2}{*}{ F4 } & Particular & 21.31 & 3.67 & $2.336 *$ & .23 \\
\hline & Nacional & 20.49 & 3.55 & & \\
\hline \multirow[t]{2}{*}{ F5 } & Particular & 19.44 & 3.17 & $3.582 * * *$ & .34 \\
\hline & Nacional & 18.29 & 3.49 & & \\
\hline \multirow[t]{2}{*}{ Total } & Particular & 177.96 & 20.03 & $2.445^{*}$ & .24 \\
\hline & Nacional & 173.48 & 17.55 & & \\
\hline
\end{tabular}

$* \mathrm{p}<.05 ; * * \mathrm{p}<.01 ; * * * \mathrm{p}<.001$ 
Con relación al colegio de procedencia, se puede apreciar que los estudiantes que provienen de colegios privados presentan actitudes más favorables que aquellos estudiantes de colegios nacionales $(\mathrm{t}(430)=$ 2.445; $\mathrm{p}<.05 ; \mathrm{d}$ de Cohen =.24). Del mismo modo, se aprecian diferencias estadísticamente significativas a favor de los alumnos de colegios particulares en las subescalas Valoración de Capacidades y Limitaciones $(\mathrm{t}(430)=4.151 ; \mathrm{p}<.001 ; \mathrm{d}$ de Cohen =.40), Calificación Genérica $(\mathrm{t}(430)=2.336 ; \mathrm{p}<.05 ; \mathrm{d}$ de Cohen $=.23)$ y Asunción de Roles $(\mathrm{t}(430)=3.582 ; \mathrm{p}<.001 ; \mathrm{d}$ de Cohen =.34).

Actitudes hacia las personas con discapacidad según el contacto con personas con discapacidad

Tabla 5

Diferencias de acuerdo a la presencia de contacto con PCD respecto a los factores de la Escala

\begin{tabular}{llllll}
\hline Factor & Contacto & Media & $\begin{array}{l}\text { Desv. } \\
\text { Estándar }\end{array}$ & t(430) & d de Cohen \\
\hline \multirow{2}{*}{ F1 } & Sí & 43.43 & 6.58 & .888 & .09 \\
& No & 42.86 & 6.53 & & \\
F2 & Sí & 57.84 & 6.46 & 1.537 & .15 \\
& No & 56.79 & 7.08 & & \\
F3 & Sí & 36.57 & 4.35 & $2.585^{* *}$ & .26 \\
& No & 35.40 & 4.68 & & .15 \\
F4 & Sí & 21.29 & 3.55 & 1.534 & .15 \\
& No & 20.74 & 3.68 & & .03 \\
F5 & Sí & 18.86 & 3.57 & -.298 & .17 \\
& No & 18.96 & 3.24 & & \\
& Sí & 178.00 & 17.97 & 1.719 &. \\
& No & 174.75 & 19.63 & & \\
\hline
\end{tabular}

$* \mathrm{p}<.05 ; * * \mathrm{p}<.01 ; * * * \mathrm{p}<.001$

En lo que respecta al contacto con las personas con discapacidad, se aprecian diferencias estadísticamente significativas a favor del grupo que sí tiene contacto en la subescala Implicación Personal $(\mathrm{t}(430)=2.585 ; \mathrm{p}<$ $.01 ; \mathrm{d}$ de Cohen =.26). 


\section{Estudiantes que presentan contacto con personas con discapacidad: Aspectos descriptivos}

Tabla 6

\section{Características de la muestra que presenta contacto con PCD}

\begin{tabular}{lcc}
\hline Sexo & Frecuencia & Porcentaje \\
\hline Razón de contacto & & \\
Familiar & 63 & 39.1 \\
Laboral & 2 & 1.2 \\
Asistencial & 1 & .6 \\
Ocio/Amistad & 69 & 42.9 \\
Otros & 26 & 16.1 \\
& & \\
Frecuencia de contacto & & \\
Muy frecuente & 23 & 14.3 \\
Frecuente & 47 & 29.2 \\
Poco frecuente & 73 & 45.3 \\
Casi nula & 18 & 11.2 \\
& & \\
Calidad de Contacto & & \\
Muy Positivo & 45 & 28.0 \\
Positivo & 75 & 46.6 \\
Neutral & 32 & 19.9 \\
Negativo & 6 & 3.7 \\
Muy Negativo & 3 & 1.9 \\
Tipo de Discapacidad & & \\
Física & & \\
Auditiva & 47 & 29.2 \\
Visual & 19 & 11.8 \\
Intelectual & 13 & 8.1 \\
Múltiple & 68 & 42.2 \\
\hline & 14 & 8.7 \\
\hline
\end{tabular}

Entre los aspectos descriptivos que pueden destacarse, la mayoría de las personas que contestaron el cuestionario y tienen contacto con personas con discapacidad, este es con personas de su entorno, sean familiares y amigos (82\%). Además de ello, un porcentaje significativo $(74.6 \%)$ calificó positivamente su contacto con las personas con discapacidad. Por otro lado, con relación a la frecuencia y calidad de contacto, se obtuvo una correlación positiva y significativa $(\mathrm{Rho}=.357 ; \mathrm{p}<.01)$, lo cual indica que mientras haya mayor contacto, este es calificado como positivo.

\section{DISCUSIÓN DE RESULTADOS}

El objetivo del presente trabajo fue describir las actitudes de los escolares limeños hacia las personas con discapacidad en función de variables tales como sexo, contacto con personas con discapacidad y tipo de colegio, debido a que la escuela es un elemento importante para la preparación de cara al empleo, la independencia y la participación en el contexto, y además el desarrollo de la integración en el ámbito escolar contribuye a transformar las actitudes sociales y profesionales hacia la población más desfavorecida (Verdugo, 2003), lo que traería consecuencias positivas a largo plazo.

Puede destacarse que en términos generales los estudiantes encuestados reportan una actitud global favorable hacia la discapacidad, debe destacarse el hecho de que la mayoría de los encuestados no se guían por prejuicios ni estereotipos con relación a las personas con discapacidad. En lo concerniente a los derechos a la normalización y a la integración social, no sobresale en el grupo encuestado. En tal sentido, puede decirse que las creencias pueden ser favorables hasta cierto punto, pero los escolares actuarían de modo contrario a lo que piensan. Esta aparente contradicción también puede apreciarse en los hallazgos de Vignes et al. (2009), quienes encontraron que el componente cognitivo obtuvo puntuaciones más baja que los componentes afectivo y conductual en la muestra estudiada.

Del mismo modo, los hallazgos no coinciden con García \& Hernández (2011), quienes reportan que sus encuestados tienden a respetar el derecho de las personas con discapacidad en temas de índole personal y social, aunque no valoran las capacidades de las personas con discapacidad.

Por otro lado, el primero de los objetivos estuvo en función de la comparación de las dimensiones de las actitudes hacia las personas con discapacidad intelectual con relación al sexo. Se encontró que las mujeres presentan, en general, actitudes más favorables, reconocen más los derechos de las personas con discapacidad, y a la par de ello se muestran más involucradas con ellos en actividades. Este aspecto es coincidente con Vignes et al. (2009) y Muratori et al. (2010), para quienes las mujeres son capaces de 
reconocer con mayor facilidad los derechos de las personas con discapacidad y tienden más a implicarse en actividades personales o sociales con ellos. No obstante, no coinciden con los hallazgos de Muratori et al. (2010), ya que las adolescentes argentinas están más desprovistas de prejuicios que sus pares varones, algo que no se aprecia en las participantes de este trabajo, quienes no difieren de sus pares varones. Aunque García \& Hernández (2011) no hallaron diferencias estadísticamente significativas respecto al sexo, esto pudo ser a causa de la variabilidad de su muestra, la cual incluyó adolescentes y adultos.

El segundo de los objetivos fue determinar si existían diferencias estadísticamente significativas con relación al colegio de procedencia, y se apreció que los alumnos de colegios particulares presentan opiniones favorables con respecto a las potenciales capacidades de las personas con discapacidad. Del mismo modo, no usan estereotipos para calificarlos, y no asumen posturas a priori acerca de su comportamiento. Este es un aspecto a destacar, dado que es rescatable que las ideas de igualdad estén presentes en los escolares, mas si estas no son canalizadas hacia algo positivo, aquellas actitudes personales, serán subsumidas por las actitudes sociales desfavorables que sobresalen en nuestro contexto, que ocasiona creencias fomentadas por aspectos culturales, históricos y sociales (Daruwalla \& Darcy, 2005), debido a que no se da la aceptación completa de las personas con discapacidad no ocurre y las actitudes negativas crean barreras para el cumplimiento de sus metas (Antonak \& Livneh, 2000).

El tercer objetivo planteado estuvo en función de explorar las diferencias en cuanto a las actitudes entre personas que tienen contacto con personas con discapacidad y aquellas que no lo tienen. No se encontraron diferencias estadísticamente significativas en cuanto a las actitudes de forma global, pero quienes mantienen contacto con las personas con discapacidad, presentan mayor implicación en cuanto a actividades conjuntas, lo que coincide con los hallazgo de Muratori et al. (2010). Esto último se sustenta en que la mayoría de los encuestados que tienen contacto con personas con discapacidad lo tienen con personas de su entorno, sean familiares y amigos (82\%), y conforme a lo que sostienen Vignes et al. (2009) y García \&
Hernández (2011), las actitudes favorables se asocia con conocimiento de discapacidad, tener un familiar cercano con discapacidad y contar con un amigo con discapacidad.

Siguiendo con este punto, se obtuvo una correlación positiva y estadísticamente significativa entre la frecuencia y calidad de contacto, lo cual indica que mientras haya mayor contacto, este es calificado como positivo, lo cual difiere de lo reportado por García \& Hernández (2011) para quienes las personas cuyo contacto con personas con discapacidad es esporádico, presentan actitudes más favorables.

Cabe mencionar que los tamaños del efecto hallados indican relevancia en las comparaciones realizadas, por lo cual los hallazgos presentados en este estudio tendrían algún tipo de significación práctica para líneas futuras de investigación.

En base a los hallazgos presentados, puede destacarse que si bien las actitudes son favorables, aquellos puntos necesarios para una completa integración, es decir, la Implicación Personal y Reconocimiento de Derechos, no se encuentran desarrollados de manera óptima, ya que si bien las creencias indican apertura con relación a la convivencia, las acciones concretas a favor de ello, aún son distantes.

Entonces, considerando que un elemento crucial es la interacción de la persona con discapacidad con el entorno del cual forma parte (Schalock \& Verdugo, 2003), esto puede resultar negativo a largo plazo, ya que son las personas que no tienen discapacidad quienes más adelante les brindarán aquellas oportunidades de integración social que les corresponden y que son necesarias para su crecimiento (Antonak \& Livneh, 2000; Moreno et al., 2006; Oliver, 1990). Esto puede ser, coincidiendo con Talou et al. (2008/2009), debido a la escasa información que se imparte sobre las personas con discapacidad en el contexto educativo, por lo cual es una tarea pendiente divulgar información en temas de discapacidad en las instituciones educativas.

$\mathrm{Si}$ bien es cierto son hallazgos preliminares, siendo la adolescencia periodo crítico en la formación de actitudes, estos primeros resultados obtenidos 
en escolares limeños son importantes debido a que permiten conocer un poco más acerca de las creencias y acciones hacia las personas con discapacidad por parte de los alumnos, y así poder contribuir más adelante a formar un país con menos desigualdades, donde exista el respeto a la diversidad y una verdadera integración, y además que por lo cual una intervención temprana resultaría favorable.

La limitación principal del estudio se refiere a la muestra, la cual fue obtenida mediante un muestreo intencional y por tanto no es representativa, por lo cual los hallazgos presentados no pueden generalizarse a la población, y en vista de ello se sugiere ampliar la muestra y extender el alcance a otros grupos, como niños y adolescentes de menor edad.

Otra de las limitaciones del presente estudio es el trabajo con encuestas. Si bien es una técnica recomendada, es probable que esté involucrada la deseabilidad social en el proceso de respuesta de los evaluados, ya que de acuerdo con Verdugo \& Arias (1991), las escalas de actitudes evalúan aquello que los individuos dicen o piensan, no lo que ellos hacen, por lo cual es fácil llegar a respuestas socialmente deseables por parte de los evaluados (Antonak \& Livneh, 2000). Ante ello, se recomienda trabajar con escalas de deseabilidad social anexas a la escala principal.

\section{REFERENCIAS}

Ajzen, I. \& Fishbein, M. (1980). Understanding attitudes and predicting social behavior. New York: Prentice Hall.

Antonak, R. \& Livneh, H. (2000). Measurement of attitudes towards persons with disabilities. Disability and Rehabilitation, 22(5), 211-224.

Daruwalla, P. \& Darcy, S. (2005). Personal and societal attitudes to disability. Annals of Tourism Research, 32(3), 549-570.

Dominguez, S. (En prensa). Tamaño del efecto: Cálculo mediante un módulo en Visual Basic. Revista de Investigación en Psicología.

Dominguez, S., Aquije, D.,Castillo, A., Dulanto, N., Llontop, C. \& Rimachi, M. (En prensa). Propiedades psicométricas de la Escala de
Actitudes hacia las Personas con Discapacidad en una muestra de escolares de Lima Metropolitana. Revista Peruana de Psicología y Trabajo Social.

García, G. \& Hernández, S. (2011). Actitudes hacia la discapacidad de jóvenes y adultos de Chiapas. Universitas Psychologica, 10(3), 817-827.

Hernández, R., Fernández, C. \& Baptista, P. (2003). Metodología de la investigación (3 Edición). México D.F.: McGraw Hill.

Imrie, R. (1997). Rethinking the relation ships between disability, rehabilitation and society. Disability and Rehabilitation, 19, 263-271.

Miller, W. G. (2003) OpenStat 4 [programa informático] [en línea], recuperado en: http:// www.statprograms 4 u.com/OpenStat_Features. htm

Montero, I. \& León, O. (2007). A guide for naming research studies in Psychology. International Journal of Clinical and Health Psychology, 7, 847-862.

Moreno, F., Rodríguez, I., Saldaña, D. \& Aguilera, A. (2006). Actitudes ante la discapacidad en el alumnado universitario matriculado en materias afines. Revista Iberoamericana de Educación, 40(5), 1-12.

Muratori, M., Guntín, C. \& Delfino, G. (2010). Actitudes de los adolescentes hacia personas con discapacidad: un estudio con alumnos de polimodal en la zona norte del conurbano bonaerense. Revista de Psicología UCA, 6(12), 39-56.

Oliver, M. (1990). The politics of disablement. Londres: Macmillan

Organización Mundial de la Salud (2001). Clasificación Internacional del Funcionamiento, de la Discapacidad y de la Salud CIF. Ginebra: Autor.

Polo, T., Fernández, C. \& Díaz, C. (2011). Estudio de las actitudes de estudiantes de Ciencias Sociales y Psicología: relevancia de la información y contacto con personas discapacitadas. Universitas Psychologica, 10(1), 113-123.

Safilios-Rothschild, C. (1976). "Disabled Persons" Self-Definitions and Their Implications for Rehabilitation. En Albrecht, G. (Ed.). The Sociology of Physical Disability and Rehabilitation 
(pp. 39-56). Pennsylvania, University of Pittsburg Press.

Schalock, R. \& Verdugo, M. (2003). Quality of life for human service practitioners. Washington, DC: American Association on Mental Retardation. [Traducido al Castellano por M.A. Verdugo y C. Jenaro. Calidad de vida. Manual para profesionales de la educación, salud y servicios sociales. Madrid: Alianza Editorial].

Shriver, T. (2003). Estudio Multinacional de Actitudes hacia las personas con discapacidad intelectual. Resultados Generales y Llamada a la acción (Informe del Comité de Olimpiadas Especiales). Washington/Boston: Special Olimpics/Center for Social Development and Education.

Talou, C., Borzi, S., Sánchez, M., Iglesias, M. \& Hernández, V. (2008/2009). La educacion de los niños y niñas com diversidad funcional desde la perspectiva de sus pares y docentes. Revista de Psicología, 10, 249-260.

Vera, A. (1995). Actitudes sociales hacia los retrasados mentales en el contexto de la integración escolar. Anales de Psicología, 11(1), 35-46.

Verdugo, M. (2003). De la segregación a la inclusión escolar. Comunicación presentada a las I Jornadas Nacionales sobre Formación y Empleo, Asturias. Recuperado el 04.05.13 desde: campus.usal.es/ inico/publicaciones/segregacion.pdf.pdf

Verdugo, M. (2005). Entrevista telefónica a Infocop On-Line. Recuperado desde: http://www.infocop. es/view_article.asp?id=465, el $03.12 .12, \quad 4: 00$ p.m.

Verdugo, M. \& Arias, B. (1991). Evaluación y modificación de las actitudes hacia los minusválidos. Revista de Psicología General y Aplicada, 44(1), 95-102.

Verdugo, M., Jenaro, C. Arias, B. (1994). Actitudes hacia las personas con discapacidad. Madrid, Ministerio de Asuntos Sociales, Instituto Nacional de Servicios Sociales.

Verdugo, M., Jenaro, C. Arias, B. (1995). Actitudes sociales y profesionales hacia las personas con discapacidad: estrategias de evaluación e intervención. En Verdugo, M. \& Aguado, A. (Dir.). Personas con discapacidad. Perspectivas psicopedagógicas y rehabilitadoras (pp. 79-143). México: Siglo XXI.

Verdugo, M., Jenaro, C. Arias, B. (2002). Actitudes sociales y profesionales hacia las personas con discapacidad: estrategias de evaluación e intervención. En Verdugo, M. (Dir.). Personas con discapacidad. Perspectivas psicopedagógicas y rehabilitadoras (pp. 79-135). Madrid: Siglo XXI.

Vignes, C., Godeau, E., Sentenac, M., Coley, N., Navarro, F., Grandjean, H. \& Arnaud, C. (2009). Determinant's of student's attitudes towards peers with disabilities. Developmental Medicine \& Child Neurology, 51, 473-479.

Fecha de recepción: 9 de agosto 2013

Fecha de aceptación: 25 de septiembre 2013 


\section{ANEXO \\ CUESTIONARIO DE OPINIONES}

INSTRUCCIONES

En la encuesta que presentamos a continuación se utiliza el término de Personas con Discapacidad para referirnos de manera general a todas aquellas personas que presentan alguna deficiencia, discapacidad o minusvalía.

Las discapacidades más comunes son: las deficiencias físicas, las deficiencias auditivas, las deficiencias visuales, el retraso mental, y la mezcla en la misma persona de alguna de esas deficiencias.

Quedan excluidas de la opinión que brindarás aquellas personas cuya discapacidad es producto de su elevada edad (los ancianos).

Su tarea consiste en opinar si está de acuerdo o no con cada una de las frases que se le van a presentar, teniendo en cuenta que:

(a) No existen respuestas buenas o malas; cada opción indica simplemente una diferente forma de pensar.

(b) Procure contestar a todas las frases, incluso a aquellas que no se ajusten a sus circunstancias concretas.

(c) En caso de duda entre varias opciones, señale aquella que se acerque más a su forma de pensar.

(d) Lea con atención cada frase, pero no se detenga demasiado en señalar su respuesta.

(e) Conteste con sinceridad. El cuestionario es totalmente confidencial y anónimo.

(f) Marque con una equis (X) su respuesta.

Los significados de las opciones son los siguientes:

Estoy totalmente de acuerdo (TA)

Estoy de acuerdo (A)

Estoy parcialmente de acuerdo (PA)

Estoy parcialmente en desacuerdo (PD)

Estoy en desacuerdo (D)

Estoy totalmente en desacuerdo (TD)

¡MUCHAS GRACIAS POR SU COLABORACIÓN!

\section{Datos personales:}

Edad:

Sexo: Masculino - Femenino

Año de estudios:

Tiene algún tipo de contacto con personas con discapacidad? SI - NO

En caso afirmativo, señale por favor:

Razón de contacto: Frecuencia de contacto: Calidad de contacto: Tipo de discapacidad:

Familiar

Labora

Asistencial

Ocio/Amistad

Otras razones
Muy frecuente

Frecuente

Poco frecuente

Casi nula
Muy Positivo:

Positivo:

Neutral:

Negativo:

Muy Negativo:
Física

Auditiva

Visual

Retraso mental

Múltiple 
1. Las personas con discapacidad con frecuencia son menos inteligentes que las demás personas.

2. Un trabajo sencillo y repetitivo es el más apropiado para las personas con discapacidad.

3. Permitiría que mi hijo aceptase la invitación a un cumpleaños que le hiciera un niño con discapacidad

4. En el trabajo, una persona con discapacidad sólo es capaz de seguir instrucciones simples.

5. Me disgusta estar cerca de personas que parecen diferentes, o actúan de forma diferente.

6. Las personas con discapacidad deberían vivir con personas afectadas por el mismo problema.

7. Las personas con discapacidad funcionan en muchos aspectos como los niños.

8. De las personas con discapacidad no puede esperase demasiado.

9. Las personas con discapacidad deberían tener las mismas oportunidades de empleo que cualquier otra persona

10. Las personas con discapacidad deberían mantenerse apartadas de la sociedad.

11. No me importa trabajar junto a personas con discapacidad.

12. Las personas con discapacidad también pueden divertirse con las demás personas.

13. Las personas con discapacidad tienen una personalidad tan equilibrada como cualquier otra persona.

14. Las personas con discapacidad también pueden casarse si lo desean.

15. Las personas con discapacidad deberían ser encerradas en instituciones especiales.

16. Muchas personas con discapacidad pueden ser profesionales competentes.

17. A las personas con discapacidad se les debería impedir votar.

18. Las personas con discapacidad a menudo están de mal humor.

19. Las personas con discapacidad confían en sí mismas tanto como las personas normales.

20. Generalmente las personas con discapacidad son sociables.

21. En el trabajo, las personas con discapacidad se entienden sin problemas con el resto de los trabajadores.

22. Sería apropiado que las personas con discapacidad trabajaran y vivieran con personas normales

23. A las personas con discapacidad se les debería prohibir pedir créditos o préstamos.

24. Las personas con discapacidad generalmente son desconfiadas.

25. No quiero trabajar con personas con discapacidad.

26. En situaciones sociales preferiría no encontrarme con personas con discapacidad.

27. Las personas con discapacidad pueden hacer muchas cosas tan bien como cualquier otra persona.

28. La mayoría de las personas con discapacidad están resentidas con las personas físicamente normales.

29. La mayor parte de las personas con discapacidad son poco constantes.

30. Las personas con discapacidad son capaces de llevar a cabo una vida social normal.

31. Si tuviera un familiar cercano con discapacidad, evitaría comentarlo con otras personas.

32. La mayor parte de las personas con discapacidad están satisfechas de sí mismas.

33. La mayor parte de las personas con discapacidad sienten que son tan valiosas como cualquiera.

34. La mayoría de las personas con discapacidad prefieren trabajar con otras personas que tengan su mismo problema.

35. Se debería prevenir que las personas con discapacidad tuvieran hijos.

36. Las personas con discapacidad son en general tan conscientes como las personas normales.

37. Deberían existir leyes que prohibieran casarse a las personas con discapacidad.

\begin{tabular}{|c|c|c|c|c|c|}
\hline TA & A & PA & PD & D & TD \\
\hline TA & A & PA & PD & D & TD \\
\hline TA & A & PA & PD & D & TD \\
\hline TA & A & PA & PD & D & TD \\
\hline TA & A & PA & PD & D & TD \\
\hline TA & A & PA & PD & D & TD \\
\hline TA & A & PA & PD & D & TD \\
\hline TA & A & PA & PD & D & $\mathrm{TD}$ \\
\hline TA & A & PA & PD & D & TD \\
\hline TA & A & PA & PD & D & $\mathrm{TD}$ \\
\hline TA & A & PA & PD & D & $\mathrm{TD}$ \\
\hline TA & A & PA & PD & D & TD \\
\hline TA & A & PA & PD & D & TD \\
\hline TA & A & PA & PD & D & $\mathrm{TD}$ \\
\hline TA & A & PA & PD & D & TD \\
\hline TA & A & PA & PD & D & $\mathrm{TD}$ \\
\hline TA & A & PA & PD & D & $\mathrm{TD}$ \\
\hline TA & A & PA & PD & D & $\mathrm{TD}$ \\
\hline TA & A & PA & PD & D & TD \\
\hline TA & A & PA & PD & D & TD \\
\hline TA & A & PA & PD & D & $\mathrm{TD}$ \\
\hline TA & A & PA & PD & D & $\mathrm{TD}$ \\
\hline TA & A & PA & PD & D & $\mathrm{TD}$ \\
\hline TA & A & PA & PD & D & $\mathrm{TD}$ \\
\hline TA & A & PA & PD & D & $\mathrm{TD}$ \\
\hline TA & A & PA & PD & D & $\mathrm{TD}$ \\
\hline TA & A & PA & PD & D & $\mathrm{TD}$ \\
\hline TA & A & PA & PD & D & TD \\
\hline TA & A & PA & PD & D & $\mathrm{TD}$ \\
\hline TA & A & PA & PD & D & $\mathrm{TD}$ \\
\hline TA & A & PA & PD & D & $\mathrm{TD}$ \\
\hline TA & A & PA & PD & D & TD \\
\hline TA & A & PA & PD & D & TD \\
\hline TA & A & PA & PD & D & $\mathrm{TD}$ \\
\hline TA & A & PA & PD & D & $\mathrm{TD}$ \\
\hline TA & A & PA & PD & D & $\mathrm{TD}$ \\
\hline TA & A & PA & PD & D & TD \\
\hline
\end{tabular}

